\title{
Fluidic microstructuring of alginate hydrogels for the single cell niche $\dagger$
}

\author{
Thomas Braschler, $\$ *$ Ana Valero, $\$$ Ludovica Colella, Kristopher Pataky, Juergen Brugger and Philippe Renaud
}

\author{
Received 9th April 2010, Accepted 3rd August 2010 \\ DOI: $10.1039 / \mathrm{c004988c}$
}

Controlling alginate gel formation by diffusion of $\mathrm{Ca}^{2+}$ ions through a filter barrier, a layer-by-layer deposition technique with resolution on the size scale of a single cell is presented. It offers the possibility of exposing cells under biocompatible conditions to microheterogeneous three-dimensional environments, mimicking the layered structure of extracellular matrix in tissues.

\section{Introduction}

Microfabrication techniques and microfluidics are increasingly being used in single-cell research. Surface patterning of cell adhesive and non-fouling patches, ${ }^{1-3}$ for instance, can confine cells to arbitrary shapes on two-dimensional surfaces, ${ }^{4}$ whereas laminar flow can be used to deliver pharmacological agents to well-defined parts of cells. ${ }^{5}$ Together with the powerful tools available through molecular biology, the control of the cellular environment at a size scale equal to or smaller than the cells is currently revealing intriguing details about the cytoskeleton mechanics, ${ }^{6,7}$ transduction schemes, ${ }^{8}$ apoptosis, ${ }^{9}$ motility ${ }^{10}$ and differentiation. ${ }^{11}$ The natural environment of mammalian cells, however, is three-dimensional rather than two-dimensional, and usually much softer than the silicon, glass or plastic surfaces typically used for cell culture experiments. For this reason, a strong research effort has gone into the development of artificial three-dimensional analogs of extracellular matrix, ${ }^{12}$ and into techniques to pattern them. Major approaches are the use of photolithographic structuring of polyethylene glycol (PEG) hydrogels, ${ }^{13}$ and fluidic layer-by-layer deposition using the natural contraction of collagen. ${ }^{14}$ The use of UV radiation in the case of PEG hydrogels in the presence of cells, and the fixed layer height given by the contraction fraction of the collagen are the major drawbacks of these two approaches. In addition, it is difficult to precisely engineer the single cell environment using these techniques. We propose on-chip layer-by-layer deposition of chemically modified alginates to achieve three-dimensional environments on the single cell scale. In the past, we have demonstrated layer-by-layer deposition of alginates with different particle loads, ${ }^{15,16}$ here we extend the technique to chemically modified alginates, enabling structuring of the environment around single cells. We provide a proof of principle of this concept by depositing alternatively fluorescently labeled and non-fluorescent alginate under biocompatible conditions; we investigate the maximum resolution achievable and show that is

Laboratory of Microsystems, Ecole Polytechnique Fédérale de Lausanne (EPFL),CH, 1015 Lausanne, Switzerland. E-mail: thomas.braschler@, epfl.ch; Fax: +41 (0)21693 59 50; Tel: +41 (0)216936582

$\dagger$ Electronic supplementary information (ESI) available: The electronic supporting material has 4 sections: (1) gel growth kinetics at different fluid flow rates; (2) theoretical estimation of the resolution of the layer-by-layer technique; (3) coupling alginate to aminofluorescein and (4) measurement of elastic modulus. See DOI: 10.1039/c004988c

\$ These authors contributed equally. well below the typical size of mammalian cells when the necessary experimental precautions are taken.

\section{Materials and methods}

\section{Design and fabrication of the microfluidic chip}

The microfluidic device is made from SU-8 structured on float glass. It consists of a channel network permitting the sequential injection of the reaction partners into a main reaction chamber (Fig. 1). The main reaction chamber is separated into an alginate and calcium side by a filter barrier, fabricated using a sacrificial layer technique based on the deposition and structuring of amorphous silicon prior to SU-8 photolithography. Both on the alginate and the calcium side we need to be able to inject a predefined sequence of pure liquids. To do so, we use a channel geometry termed "virtual valve" developed in our laboratory. ${ }^{16}$ Here, two virtual valves are used, each consisting of 4 channels converging towards the calcium or alginate side of the reaction chamber respectively (Fig. 1A). A combination of controlled backflow and bypass channels allows to avoid cross-contamination between the inlets, ${ }^{16}$ while selecting an arbitrary sequence among the 4 inlets on each side. The gel formation is initiated by

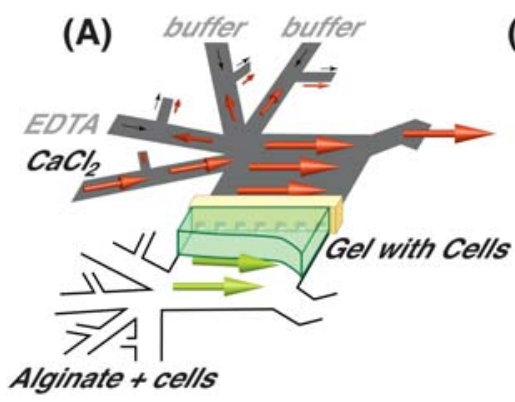

(B)

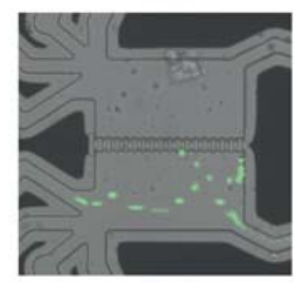

Fig. 1 (A) Schematic of the microfluidic device used for gel growth with the corresponding inlets for loading. The reaction chamber is divided into two zones by a filter barrier: the calcium (upper) and the alginate side (bottom). On the alginate side, a gel will be formed by the reaction of free alginate with $\mathrm{Ca}^{2+}$ ions that diffuse through the filter barrier. For switching among the different liquids on either side, virtual valves are used. The virtual valves use bypass channels in combination with the flow pattern illustrated exemplarily for the calcium side to keep all inlets clean while allowing to select an arbitrary sequence among them. ${ }^{16}$ (B) Micrograph of the fluidic device for gel formation. Immobilized cells are stationary, while the liquid alginate precursor solution is still flowing. We use EG7 cells stained with fluorescein diacetate, showing their viability. 
selecting a $\mathrm{CaCl}_{2}$ solution on the calcium side of the filter and a sodium alginate precursor solution on the other, since the $\mathrm{Ca}^{2+}$ ions will diffuse across the filter to crosslink the alginate on the other side. The filter barrier avoids gel rupture due to uncontrolled pressure differences. By sequentially switching alginate types, different layers are deposited.

\section{Chemicals}

Alginate, MES (2-( $N$-morpholino)ethanesulfonic acid) buffer, Tris (tris(hydroxymethyl)-aminomethane), EDC (1-ethyl-3-[3dimethylaminopropyl] carbodiimide hydrochloride), $\mathrm{HCl}$, $\mathrm{NaOH}, \mathrm{NaCl}, \mathrm{CaCl}_{2}$, EDTA, and aminofluorescein were all obtained from Sigma-Aldrich (Buchs, Switzerland). Fluorescently labeled alginate is synthesized by activation of the carboxylate groups of the alginate by EDC followed by coupling to the amino-group of aminofluorescein. The protocol is a simplified variant of the labeling protocol developed by Strand et al. ${ }^{17}$ the details are given in the ESI $3 \uparrow$. Generally, the solution are supplemented with $150 \mathrm{mM} \mathrm{NaCl}$ and $100 \mathrm{mM}$ Tris- $\mathrm{HCl}, \mathrm{pH}$ 9; the alginate solutions used for gel construction are $1 \% \mathrm{w} / \mathrm{w}$. Solutions containing mammalian cells are prepared using the cell culture medium (see section Cell culture below) plus alginate 1\% $\mathrm{w} / \mathrm{w}$ sodium alginate; in experiments involving cells, no Tris- $\mathrm{HCl}$ is used.

\section{Cell culture}

EG7 cells, a non-adherent $\mathrm{T}$-cell lymphoma cell line, were cultured in an incubator $\left(5 \% \mathrm{CO}_{2}, 37^{\circ} \mathrm{C}\right)$ as a suspension culture in RMPI 1640 medium containing Glut Amax (Invitrogen, Switzerland), supplemented with $10 \%$ fetal bovine serum (FBS), penicillin-streptomycin, $50 \mu \mathrm{M} \beta$-mercaptoethanol (all from Gibco, Switzerland) and $400 \mu \mathrm{g} \mathrm{ml}{ }^{-1}$ G418-sulfate solution (Brunschwig).

\section{Cell viability}

Viability of the immobilized cells is assessed with fluorescein diacetate; EG7 cells suspended in RPMI+ medium $\left(0.5 \times 10^{6}\right.$ cells $\mathrm{ml}^{-1}$ ) were mixed with fluorescein diacetate (Fluka, Buchs,
Switzerland) to obtain a final concentration of $50 \mu \mathrm{g} \mathrm{ml}^{-1}$ during an incubation time of $15 \mathrm{~min}$. Cells were washed by spinning down and resuspending in fresh medium. Fluorescein-diacetate is cleaved by intracellular esterases, and the resulting hydrophilic fluorescein is unable to leave intact cells. So accumulation to high levels indicates the presence of both intracellular enzymatic activity and membrane integrity, ${ }^{18,19}$ and hence, cell viability.

\section{Image acquisition and analysis}

The microfluidic device was mounted onto an XYZ translation stage in an inverted microscope (Leica DMIL, Leica Microsystems, Wetzlar, GmbH, Germany). The microscope system is equipped with a mercury lamp, $10 \times, 20 \times$ and $40 \times$ objectives, and the fluorescence filter set BP 450-490. In addition, a computer-controlled camera (uEye) is mounted in the microscope for image/video recording.

\section{Assessment of the resolution of the layer-by-layer technique}

The resolution of the layer-by-layer gel construction technique is quantified by depositing alternating layers of fluorescently labeled and non-fluorescent alginate and measuring the contrast between the alternate layers. The contrast is defined as:

$$
\text { contrast }=(\max (I)-\min (I)) / \max (I)
$$

$I$ being the fluorescence intensity, and the maxima and minima being measured on adjacent layers.

\section{Results and discussion}

\section{Deposition of chemically distinct layers}

By changing the alginate precursor solution during the gel growth using the virtual valve,${ }^{16}$ we get successive distinct layers. If the alginate is chemically modified, successive layers of chemically distinct alginate are obtained. This concept is illustrated in Fig. 2. Fig. $2 \mathrm{C}$ shows an actual fluorescence image of the layers obtained. The shape of the layers is essentially determined by the diffusion of $\mathrm{Ca}^{2+}$ ions, leading to bands that are parallel to the filter structure. Close to the edges, however, additional effects

\section{(A) First Gel Layer}

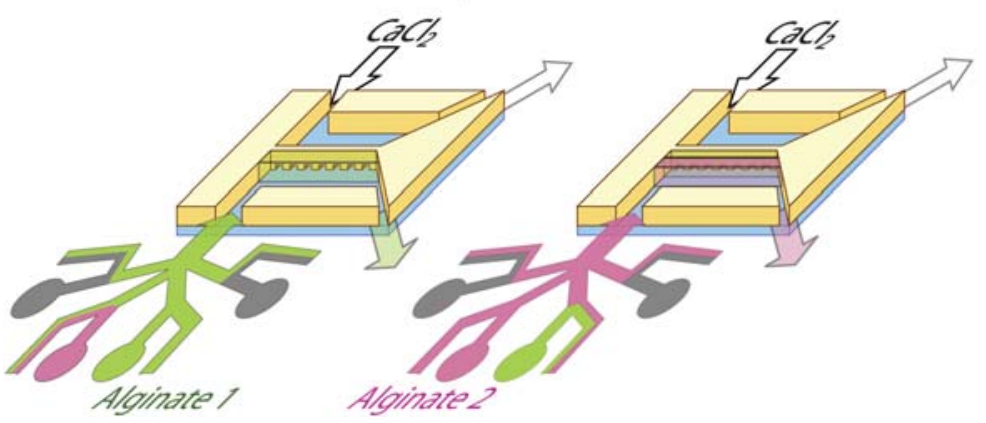

\section{$\begin{array}{lll}\text { (B) Second Gel Layer } & \text { (C) Experimental Layer Growth }\end{array}$}

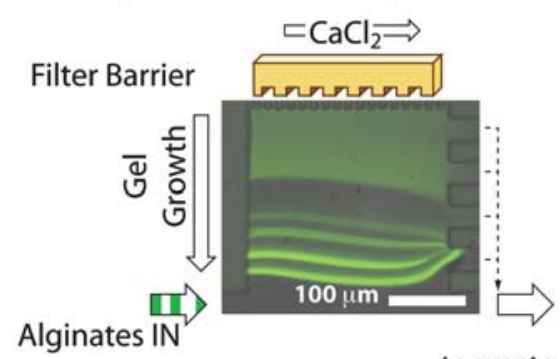

to waste

Fig. 2 Principle of sequential gel layer construction. By switching the alginate precursor during the gel growth, layers of chemically modified alginate are grown (A) and (B). In (C), gel layers are constructed by sequentially applying aminofluorescein labeled alginate alternating with non-fluorescent alginate; between sequential alginate growth phases buffer is used to flush out the previous alginate. The micrograph is an overlay of a gray scale image of the chip taken under white light illumination and the layers taken under fluorescence illumination. The filter is visible at the top edge of the micrograph, and the gel formation chamber extends to the bottom edge of the micrograph. 
of the fluid flow can be seen: rapid flow of the alginate precursor along the gel boundary slows down the gel growth. By detailed investigation of the gel growth rates at different fluid flow rates, we can conclude that this is most likely because of the washout of partially crosslinked alginate molecules from the reaction zone (data provided in the ESI $1 \dagger$ ).

Our next goal is to establish the limiting resolution of the layerby-layer technique. In Fig. 3 fluorescent and non-fluorescent alginate layers, grown sequentially, are shown. In Fig. 3A, the layer growth is obtained by alternate switching of alginates, which is the most direct and basic implementation of the principle outlined in Fig. 2. In Fig. 3B we intercalate a phase with buffer flow between sequential alginate growth phases. Both alginates, fluorescent and non-fluorescent labeled, have identical concentrations ( $1 \%$ by weight), whereas on the calcium side, a $100 \mathrm{mM} \mathrm{CaCl}$ solution is used (all solutions are supplemented with $150 \mathrm{mM} \mathrm{NaCl}$ and $100 \mathrm{mM}$ Tris- $\mathrm{HCl}$ as outlined in Materials and methods). The flow rate is $0.6 \mathrm{~nL} \mathrm{~s}^{-1}$, as estimated by determination of the fluid speed by tracing fluorescent polystyrene beads (data not shown). This represents a compromise where substantial gel growth and rapid exchange of the alginate precursor are still possible. To obtain layers of regular width, we first establish a gel growth curve by noting the advancement of
(A) Direct Deposition
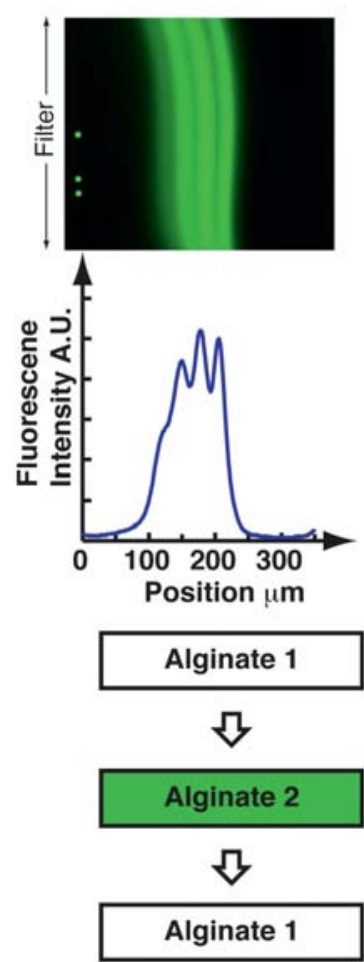

\section{(B) Intercalated Buffer}
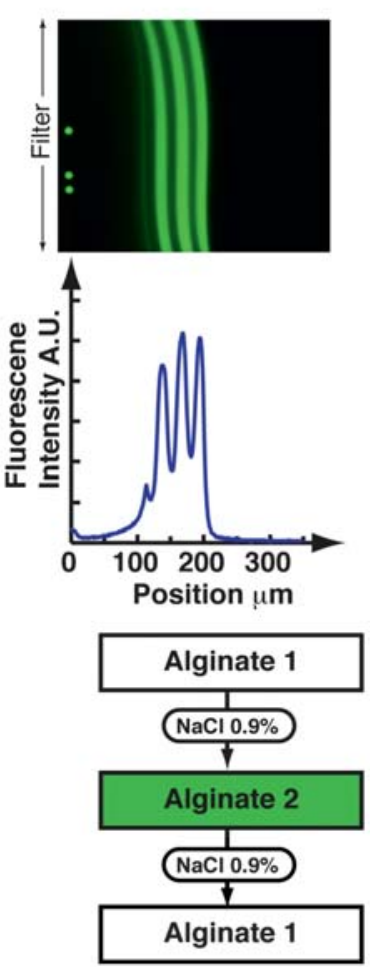

Fig. 3 Layers produced by simple switching of alginates (A) compared to layers produced by a buffer step intercalated into the switching procedure (B). Switching alginates during gel growth is critical because of concentration variations and partial mixing due to the parabolic flow profile. This leads to less than optimal layers that appear washed out (A) and have a lower contrast between adjacent layers. It is possible to use buffer between the alginate phases, allowing to wash out the old alginate more quickly because of the lower viscosity of the buffer. This limits crosscontamination as indicated by the better distinction of the layers in (B). the gel front as a function of time, and then determine the switching time points to obtain the desired layer width. In the ESI $1 \uparrow$, examples of gel growth curves are given; these gel growth curves are chip-specific, and dependent on the fluid flow-rate.

When intercalating buffer between successive alginate layers, the previous alginate present in the channel leading from the virtual valve to the reaction chambers is washed out; in addition, unreacted alginate that could otherwise diffuse into the next layer is washed out from the gel's edge. This results in more clearly defined alginate layers, and hence better resolution than if alginates were switched directly. Therefore, using buffer helps to avoid mixing of the successive alginate precursors and greatly improves the resolution of the layer-by-layer technique.

\section{Resolution of the alginate layers by sequential deposition}

We shall now provide an experimental evaluation and theoretical framework to understand the limiting factors for the resolution of the layer-by-layer technique. This will allow us to provide an improved method to indeed produce gradients on the micrometer scale.

We quantify the resolution of the layer-by-layer gel construction technique by evaluating the contrast between successive layers as defined by eqn (1) from fluorescence intensity images, for layers of a given width. To construct layers of known nominal width, we use predetermined timed switching cycles between fluorescent and non-fluorescent alginate.

Fig. 4 shows a quantitative estimation of the layers' resolution produced by both techniques; direct switching of alginates and buffer intercalation. Taking 50\% contrast as the criterion for the limiting resolution, we find $10 \mu \mathrm{m}$ as a limiting resolution for the

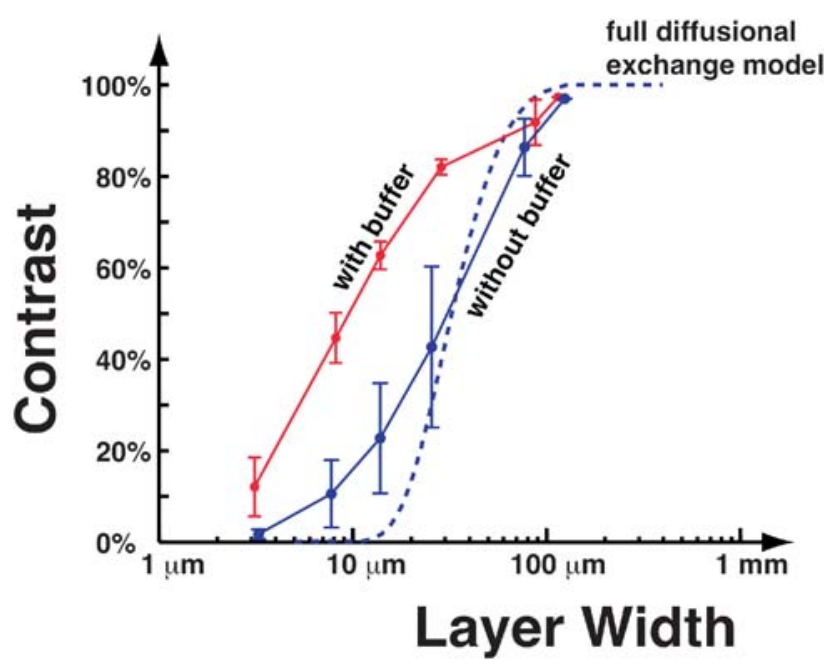

Fig. 4 Layers resolution; by measuring the photographic contrast between successive layers we assess the resolution of the layer-by-layer alginate gel growth technique. The procedure is repeated for layers of varying nominal spacing. The limiting resolution is defined by a contrast of $50 \%$ between adjacent layers. We observe about $10 \mu \mathrm{m}$ resolution when intercalating buffer between successive alginate precursor phases, and $30 \mu \mathrm{m}$ by simply switching alginates. The theoretical curve labeled "full diffusional exchange model" is obtained by evaluating the contrast based on eqn (1) and (3). The underlying hypothesis is that all mobile alginate chains are exchanged by diffusion at each switching cycle. 
method with buffer intercalation between successive alginate precursor solutions, and $30 \mu \mathrm{m}$ for the direct switching. Obviously, for the purpose of building sharp gradients at the scale of a single cell the layer-by-layer technique with buffer intercalation is more suitable than the direct alginate switching method, but it would be desirable to improve the resolution further.

\section{Resolution of the layer-by-layer technique and the reaction front structure}

The structure and in particular the width of the reaction front arising from the reaction between $\mathrm{Ca}^{2+}$ and alginate are determined by the balance between the speed of the chemical reaction between alginate and $\mathrm{Ca}^{2+}$ ions, which tends to decrease the width of the reaction zone, and the diffusion of the $\mathrm{Ca}^{2+}$ ions and the alginate molecules, which tends to increase the width of the reaction zone. By providing a simplified analytical solution to an established numerical model of the alginate reaction front, known as the Mikkelsen-Elgsaeter model, ${ }^{20,21}$ we are able to obtain a theoretical estimate for the width of the reaction zone, and hence approximatively the resolution of the layer-by-layer deposition technique if no special precautions are taken. Indeed, in the ESI $2 \dagger$, we show that the minimal characteristic spatial extent of the reaction front $W$ can be estimated by the following scaling expression:

$$
W=\sqrt{\frac{D_{\mathrm{c}}}{k a_{0}^{2} N_{\mathrm{c}}}}
$$

Using the literature values for the diffusion coefficient of the $\mathrm{Ca}^{2+}$ ions $^{22}\left(D_{\mathrm{c}}=0.78 \times 10^{-9} \mathrm{~m}^{2} \mathrm{~s}^{-1}\right)$, the reaction rate constant ${ }^{21}$ $\left(k=5000 \mathrm{M}^{-1}\right)$, and the stoichiometric $\mathrm{Ca}^{2+}$-binding capacity per alginate residue $\left(N_{\mathrm{c}} \approx 0.3\right.$ for an alginate containing $39 \%$ guluronic acid residues, ${ }^{23}$ according to eqn (11) in the ESI $2 \dagger$ ), as well as the initial bulk concentration of the alginate solution in terms of uronic acid residues $\left(a_{0}=1 \%\right.$ alginate $=50 \mathrm{mM}$ uronic acid residues since each residue has a molar mass of $200 \mathrm{~g} \mathrm{~mol}^{-1}$ for sodium alginate), we obtain an estimate of $W \approx 15 \mu \mathrm{m}$ for the alginate solution used here. Concerning the layer-by-layer deposition, in the worst case, switching alginates implies replacement of the entire population of mobile alginate molecules in the reaction zone, and hence maximal blurring between adjacent layers will occur. In this case, the resolution will be on the order of $W$. More precisely, using the hypothesis of complete replacement of the non-crosslinked alginate molecules with every switching cycle, it is possible to derive an expression for the local fluorescent alginate concentration. The details are given in the ESI $2 \uparrow$. The result is presented in eqn (3):

$$
g_{\text {fluorescent }}\left(x_{0}\right)=a_{0} \sum_{i \in Z}\left(\beta\left[\frac{x_{0}+2 i \Delta x}{W}+\frac{\Delta x}{W}\right]-\beta\left[\frac{x_{0}+2 i \Delta x}{W}\right]\right)
$$

where $x$ is the spatial coordinate, whereas $W$ is given by eqn (2). $\beta$ is a monotonically rising function defined by eqn (30) in the ESI $2 \uparrow$. Applying eqn (1) numerically to the profiles obtained by eqn (3), we obtain a theoretical estimation of the contrast as a function of the nominal layer width, as indicated by the theoretical line labeled "full diffusion exchange model" in Fig. 4.
We find that in particular for the buffer intercalation technique, the resolution in practice is better than the theoretical estimate obtained by using eqn (1) and (3), showing that the new alginate does not manage to replace the entire population of mobile molecules in the reaction zone before getting polymerized. We expect this to be because the increased viscosity of the gel hinders diffusive exchange with the adjacent liquid precursor. Furthermore, since we do not stop the $\mathrm{Ca}^{2+}$ supply during the buffer washing step, there will be $\mathrm{Ca}^{2+}$ ions available in the immediate vicinity of the gel edge, allowing for rapid attachment of the alginate molecules to the existing gel. Reduced exchange between the gel and the adjacent precursor liquid in turn is synonymous with improved resolution.

\section{Improvement of the resolution by EDTA back etching}

The best possible resolution and hence the steepest gradients would be obtained if the following three conditions are met: (1) an infinitely sharp edge when starting a new layer; (2) no diffusion of the liquid precursor into the existing gel and (3) no diffusion of partially cross-linked alginate from the previous layer into the new layer. Condition 3 is probably naturally met when intercalating a washing step between successive alginates, since this should also wash out mobile alginate chains from the reaction zone. Condition 1 is difficult to fulfill at edge of growing layer because of the finite width of the reaction zone. To improve edge sharpness, we use a dilute EDTA solution to etch back the gel. In this case, the sharpness of the gel edge is no more determined by the process of gel growth and its relatively large reaction zone, but by the chemical process of gel dissolution by EDTA. Since the reaction rate of EDTA with the $\mathrm{Ca}^{2+}$ ions is much higher than the one between alginate and $\mathrm{Ca}^{2+}$ ions, we expect a much smaller reaction zone and hence a better-defined gel edge. Finally, regarding condition 2 , avoiding the diffusion of liquid precursor into the already crosslinked gel is difficult without additional experimental measures such as applying an electric field to take advantage of electrophoretic alginate migration. However, when considering a gradient of a single active substance, this might not be necessary. Indeed, if we consider the transition from a labeled layer to a native alginate layer, then back-diffusion of non-labeled alginate into the fluorescently labeled layer is of minor importance for the fluorophore concentration gradient. Transposed to our model system (aminofluorescein label) to quantify the resolution and gradient steepness, this means that we expect the transition from a fluorescent layer to a non-fluorescent layer to be sharper than the inverse transition-provided that diffusion from the liquid precursor into the gel is indeed quantitatively much more important than diffusion of partially gelled alginate back into the liquid precursor.

Fig. 5A shows a high resolution fluorescence profile, obtained by confocal microscopy of a fluorescent layer flanked by two non-fluorescent layers, as obtained by using back etching by a dilute EDTA solution between successive layers. Fig. 5B recapitulates the experimental steps used for alternating layer growth and EDTA back-etching. It is clear that the peak in the fluorescence profile corresponding to a single layer of fluorescent alginate sandwiched between layers of native, non-fluorescent alginate is highly asymmetric. In particular, the back-etching 


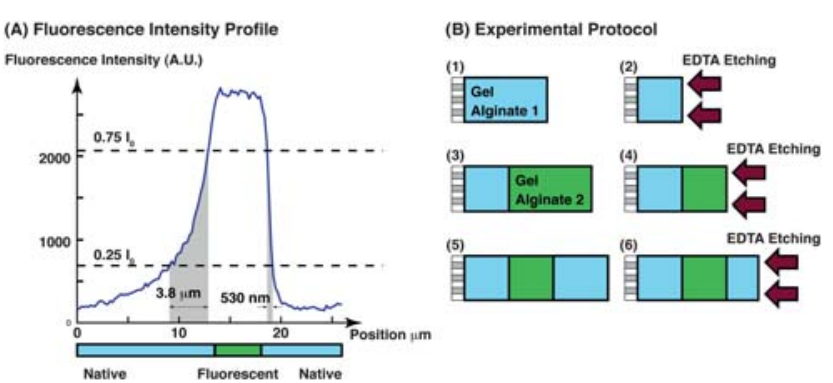

Fig. 5 Resolution limit using the EDTA back-etching technique. (A) shows the fluorescence intensity profile across a layer of fluorescent alginate flanked by two non-fluorescent layers. The sharpest gradient in terms of fluorescently labeled alginate is obtained at the falling edge, corresponding to the transition from fluorescent to non-fluorescent alginate. (B) shows schematically the protocol used. Essentially, for each layer, a thick initial layer is grown (steps 1, 3, and 5); to sharpen the layers, an intermediate EDTA back-etching step is used (steps 2, 4, and 6). The EDTA solution contains $7 \mathrm{mM}$ in addition to the $150 \mathrm{mM} \mathrm{NaCl}$ and $100 \mathrm{mM}$ Tris- $\mathrm{HCl}$ as for the other solutions.

technique affords a very steep gradient on the falling edge. The rising edge reflects diffusion of the fluorescent alginate precursor back into the existing gel, giving rise to a transition zone of several micrometers width as is expected for a process reflecting competition between alginate diffusion and reaction. The sharp falling edge reflects the sharpness of the EDTA back-etched gel edge, since back-diffusion of the following non-fluorescent alginate will not be seen. Indeed, in the steepest part of the fluorescence intensity profile, the concentration of the fluorescent label drops by a factor of 3 over no more than $530 \mathrm{~nm}$. This is close to the resolution limit of the confocal microscope employed, and in any case entirely sufficient to create gradients at scales smaller than single cells.

\section{Stability of the layers}

It is known that alginate poly-lysine capsules slowly leak out alginate, losing up to $50 \%$ of their mass in a few weeks time. ${ }^{24}$ This raises the question of how much diffusional mobility the alginate chains retain in the gelled state, since slow diffusion after layer formation would degrade the pattern. We tried to assess the loss of alginate by following the fluorescence profiles over time. Since photobleaching occurs with each exposure, we follow the relative intensity distribution rather than the absolute intensity. First, we perfused a layered alginate gel containing fluorescent and non-fluorescent layers during four days with a physiologic saline solution supplemented with $c a$. $10 \mathrm{mM} \mathrm{CaCl}_{2}$ (at rate of ca. $0.5 \mathrm{ml} \mathrm{day}{ }^{-1}$ ). The concentration indication is approximate since we used diffusive mixing on chip to generate this solution. Taking every day a picture using a few seconds exposure, we can follow eventual diffusion of the fluorescently labeled molecules inside the alginate gel. The result is shown in Fig. 6A, days 1-4. The fluorescence intensity is normalized so that the mean intensity within the original gel band remains constant, in order not to be influenced by the progressive photobleaching that diminishes the fluorescence intensity with time. We observe that the fluorescence distribution remains essentially constant during 4 days, indicating that no substantial pattern degradation takes
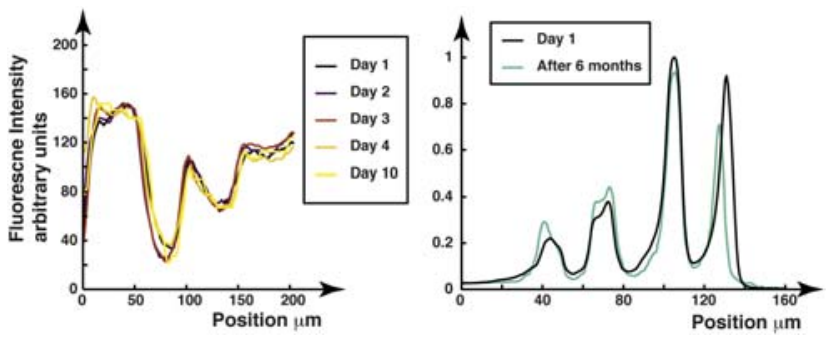

Fig. 6 Stability of the gel layers. (A) assesses the stability on a time scale of days, (B) on the time scale of months. For (A), a fluorescence profile was taken immediately, and on days 2, 3, 4 and 10 after gel construction. From day 1 to 4 we perfused the gel with a slow flow of $10 \mathrm{mM} \mathrm{CaCl}$ in $0.9 \% \mathrm{NaCl}$ to test for pattern degradation due to remaining low mobility of the alginate in the gel. After day 4, we filled the entire chip with alginate and let it polymerize such as to prevent all fluid flow next to gel. Under these conditions, we expect also to see pattern degradation due to alginate chains becoming suddenly mobile, since they would not be washed away. No significant pattern degradation can be detected under either condition on the time scale of the experiment. For (B), the chip was opened after gel construction, and the entire chip submerged in $5 \mathrm{ml} \mathrm{NaCl} 0.9 \%$. After 6 months of storage in the dark, the fluorescence intensity profile was assessed again, showing pattern stability on a time scale of months.

place in this period. There remains, however, a remote possibility that the alginate loss could occur in a binary manner - that is, alginate chains would remain fixed unless some catastrophic and random event frees them to be washed away quickly with the perfusion medium. To exclude this possibility, difficult to distinguish from photobleaching under perfusion, we blocked off the flow on day 4 by injecting a supplementary bolus of alginate and $\mathrm{CaCl}_{2}$ and letting the gelling reaction proceed until the entire chamber was blocked with alginate gel. We then left the sample for another 6 days in the dark, and quantified the distribution of the fluorescence intensity on day 10 . We detect no broadening of the intensity pattern from day 4 to day 10; this excludes the possibility of the alginate becoming mobile in a sudden fashion, on the time scale of several days.

Finally, in order to assess layer stability on a long term scale, we opened a chip with a layered gel obtained by the EDTA backetching technique and placed it for 6 months in $5 \mathrm{mM} \mathrm{CaCl}_{2}$ in physiological salt solution. After 6 months, we assessed again the fluorescence distribution. The results are shown in Fig. 6B, and indicate that the layers are stable on this time scale.

\section{Heterogeneous environments for single cells}

The resolution of the layer-by-layer technique is on the order of the diameter of typical mammalian cells, and much sharper gradients can be obtained by the EDTA back-etching technique. We can therefore use the technique to create an inhomogeneous environment at the scale of single cells. A simple possibility is to deposit two or more layers over one cell. To achieve this the following protocol can be used. The cell is first included into a thick layer of alginate gel. Then EDTA is flown along the gel on the alginate side, to etch the gel until the cell is protruding from the gel to the desired extent. At this point of time, a new alginate precursor solution is brought into contact with the gel. Again, a thick layer is grown, covering the previously protruding part of 
(A)

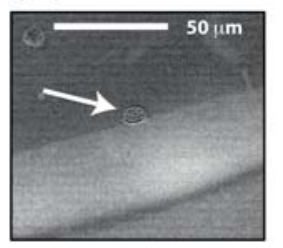

(B)

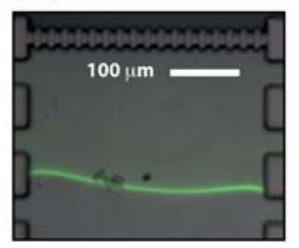

(C)

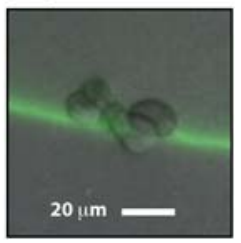

Fig. 7 Structuring of alginates on the single-cell scale. EG7-cells are included into an alternation of gel layers. (A) shows a single cell (arrow) embedded at the boundary between a non-fluorescent and a fluorescent layer. (B) and (C) show a cell group embedded at a three-layer interface: the entire held of view is filled with gel, but there is a sequence of nonfluorescent, fluorescent, and non-fluorescent gel, the small fluorescent band being centered on the cell group. (C) is a magnified view of (B).

the cell. This gives rise to a transition between alginate types at the scale of a single cell, as shown in Fig. 7A. It is possible to again etch the gel with EDTA, and deposit another layer of alginate. If the middle layer is made sufficiently thin, the cell will be in contact with three different layers. An example of a such a three-layer structure is shown in Fig. 7B and C, where three layers are deposited as follows: non-fluorescent, fluorescently labeled and non-fluorescent. This shows that we can control the chemical composition of the cell entrapment matrix with sufficient precision to engineer single cell niches.

Concerning the chip design for cell inclusion, it is advantageous to ensure that the main flow direction in the reaction chamber is parallel to the gel front. Indeed, if on the contrary, the gel is in a deep pouch, it is difficult to bring cells into the gelling region because of size exclusion effects. For this reason, we use chips with several parallel inlet and outlet channels for cell inclusion experiments.

Finally, it is known that cells are responsive, among other factors, to the stiffness of the extracellular matrix. ${ }^{25}$ Since the stiffness of alginate hydrogels depends on the $\mathrm{Ca}^{2+}$ concentration, an important question is to know whether the microfluidic deposition technique presented here leads to a gradient in elastic modulus across the gel. We investigate this question by measuring the elastic modulus for alginate gel slabs, and in particular its evolution upon changing the $\mathrm{Ca}^{2+}$ concentration (data provided in ESI $4 \uparrow$ ). The answer is that during the gel deposition, we expect the $\mathrm{Ca}^{2+}$ concentration gradient from the filter to the reaction front to cause a gradient in elastic modulus of the gel, but that this gradient disappears when the gel is incubated in a homogeneous medium. This is because the modulus of Ca-alginate hydrogels, at least in the presence of physiological $\mathrm{Na}^{+}$concentrations, is a function of the current free $\mathrm{Ca}^{2+}$ concentration, and not of the gel formation history.

\section{Conclusions}

In this report, we demonstrate the deposition of an alginatebased, structured extracellular-matrix-mimetic environment for single cells. The technique is fully biocompatible, and provides patterning and layer resolution beyond the single cell scale. It permits the precise engineering of the three-dimensional chemical environment in which the cell resides.
The true interest of being able to deposit layers at the scale of a single cell is to influence and study cellular processes such as orientation of the cell division axis, migration, asymmetric division, or morphological differentiation. This report presents a technique and setup for the creation of a controlled microheterogeneous environment for living cells. The next step is to provide biologically meaningful cues to the embedded cells, either by covalently bound peptides, ${ }^{26,27}$ growth factors entrapped in the alginate, ${ }^{28}$ or by the use of interpenetrating polymer networks with the possibility to dissolve away the alginate once the second polymer is set. ${ }^{29}$

We anticipate that this platform will be useful in the context of mimicking the natural cellular environment on the single cell or microtissue level, in important fields such as stem cell differentiation and drug discovery.

\section{Acknowledgements}

We wish to thank Andrea Negro and Dr Sammy Gobaa for their kind help with the rheology measurements, as well as Harald Van Lintel and Dr Arnaud Bertsch for proofreading the manuscript. This work was in part funded by the European Community CellPROM project under the 6th Frame-work Program, contract No. NMP4-CT-2004-500039.

\section{Notes and references}

1 J. Y. Lee, S. S. Shah, C. C. Zimmer, G. Y. Liu and A. Revzin, Langmuir, 2008, 24, 2232-2239.

2 M. Mrksich, C. S. Chen, Y. N. Xia, L. E. Dike, D. E. Ingber and G. M. Whitesides, Proc. Natl. Acad. Sci. U. S. A., 1996, 93, 10775-10778.

3 A. Revzin, R. G. Tompkins and M. Toner, Langmuir, 2003, 19, 9855-9862.

4 R. S. Kane, S. Takayama, E. Ostuni, D. E. Ingber and G. M. Whitesides, Biomaterials, 1999, 20, 2363-2376.

5 S. Takayama, E. Ostuni, P. LeDuc, K. Naruse, D. E. Ingber and G. M. Whitesides, Nature, 2001, 411, 1016.

6 Y. Tanaka, K. Morishima, T. Shimizu, A. Kikuchi, M. Yamato, T. Okano and T. Kitamori, Lab Chip, 2006, 6, 230-235.

7 M. E. Chicurel, C. S. Chen and D. E. Ingber, Curr. Opin. Cell Biol., 1998, 10, 232-239.

8 D. W. Hamilton, F. Jamshidi and D. M. Brunette, Materialwiss. Werkstofftech., 2009, 40, 101-107.

9 C. S. Chen, M. Mrksich, S. Huang, G. M. Whitesides and D. E. Ingber, Biotechnol. Prog., 1998, 14, 356-363.

10 A. Brock, E. Chang, C. C. Ho, P. LeDuc, X. Y. Jiang, G. M. Whitesides and D. E. Ingber, Langmuir, 2003, 19, 1611-1617.

11 G. J. Bakeine, L. Benedetti, D. Galli, G. Grenci, A. Pozzato, M. Prasciolu, M. Tormen and G. Cusella, Microelectron. Eng., 2010, 87, 830-833.

12 M. P. Lutolf, P. M. Gilbert and H. M. Blau, Nature, 2009, 462, 433-441.

13 A. Revzin, R. J. Russell, V. K. Yadavalli, W. G. Koh, C. Deister, D. D. Hile, M. B. Mellott and M. V. Pishko, Langmuir, 2001, 17, $5440-5447$.

14 W. Tan and T. A. Desai, Biomaterials, 2004, 25, 1355-1364.

15 T. Braschler, R. Johann, M. Heule, L. Metref and P. Renaud, Lab Chip, 2005, 5, 553-559.

16 T. Braschler, J. Theytaz, R. Zvitov-Marabi, H. Van Lintel, G. Loche, A. Kunze, N. Demierre, R. Tornay, M. Schlund and P. Renaud, Lab Chip, 2007, 7, 1111-1113.

17 B. L. Strand, Y. A. Morch, T. Espevik and G. Skjak-Braek, Biotechnol. Bioeng., 2003, 82, 386-394.

18 J. Boender, Vet. Q., 1984, 6, 236-240.

19 J. M. Widholm, Stain Technol., 1972, 47, 189.

20 A. E. Arne Mikkelsen, Biopolymers, 1995, 36, 17-41. 
21 M. M. J.-M. Duez, R. Demeure, J. F. Goudemant, B. P. Hills and J. Godward, Magn. Reson. Chem., 2000, 38, 324-330.

22 J. H. Wang, J. Am. Chem. Soc., 1953, 75, 1769-1770.

23 D. Kaplan, Biopolymers from Renewable Resources: Macromolecular Systems, Springer, 1998.

24 V. Breguet, PhD thesis, EPFL, 2007.

25 A. M. Kloxin, J. A. Benton and K. S. Anseth, Biomaterials, 2010, 31, $1-8$.
26 S. X. Hsiong, T. Boontheekul, N. Huebsch and D. J. Mooney, Tissue Eng. A, 2009, 15, 263-272.

27 J. A. Rowley, G. Madlambayan and D. J. Mooney, Biomaterials, 1999, 20, 45-53.

28 C. A. Simmons, E. Alsberg, S. Hsiong, W. J. Kim and D. J. Mooney, Bone, 2004, 35, 562-569.

29 N. P. Desai, A. Sojomihardjo, Z. Yao, N. Ron and P. Soon-Shiong, Journal of Microencapsulation, 2000, 17, 690. 